\title{
El niño obeso en la Unidad de Cuidados Intensivos. Puesta al día
}

The obese child in the Intensive Care Unit. Update

\author{
Dr. Alejandro Donoso F. ${ }^{a}$, Dr. Pablo Córdova L. ${ }^{a}$, Dra. Pilar Hevia J. ${ }^{b}$ y \\ Dra. Daniela Arriagada S. ${ }^{a}$
}

\begin{abstract}
RESUMEN
Actualmente, la obesidad infantil constituye una epidemia. La frecuencia de pacientes críticos con sobrepeso u obesidad ha aumentado rápidamente en las unidades de cuidados intensivos. El tejido adiposo es un órgano endócrino que secreta varias hormonas proteicas, entre las que se destaca la leptina, la cual es reguladora de su masa. La presencia de hipertensión arterial, síndrome metabólico, diabetes mellitus, enfermedades respiratorias y enfermedad renal crónica se puede manifestar en el paciente pediátrico obeso durante su estadía en la Unidad de Cuidados Intensivos y complicar su evolución. El tratamiento de la obesidad es complejo y debe involucrar al paciente, a su familia y a la comunidad médica. Debe realizarse en coordinación con políticas sanitarias gubernamentales integrales y en conjunto con un cambio de marco cultural.

Palabras clave: obesidad, leptina, sindrome metabólico, hipertensión, cuidados intensivos.
\end{abstract}

http:/ / dx.doi.org/10.5546/aap.2016.258

\section{INTRODUCCIÓN}

La discusión sobre la obesidad o malnutrición por exceso y su efecto deletéreo en la salud ha provocado gran interés, pero comparativamente poco éxito, en relación con las estrategias de prevención implementadas. ${ }^{1,2}$

En regiones en vías de desarrollo, como América Latina, la cual se encuentra en transición epidemiológica, existe una elevada tasa de obesidad, donde más del $90 \%$ de la obesidad infantil es primaria o exógena, causada por un excesivo consumo calórico en un ambiente con determinantes sociales, como pobreza, falta de educación y accesibilidad a los alimentos. Los casos remanentes son secundarios o "endógenos" y pueden ser ocasionados por trastornos endócrinos, defectos genéticos asociados a obesidad o enfermedades con daño hipotalámico. ${ }^{3}$
La obesidad infantojuvenil es un factor de riesgo de diversas enfermedades y está asociada con una importante morbilidad crónica (obesidad adulta) y mortalidad prematura. ${ }^{4,5}$ Se ha señalado una disminución de las expectativas de vida de entre 5 y 20 años. ${ }^{6}$

La obesidad se define por el exceso de grasa corporal (masa grasa) en relación con la talla. En la actualidad, se recomienda el uso del índice de masa corporal (IMC), el cual se calcula como el peso $(\mathrm{kg}) / \mathrm{talla}\left(\mathrm{m}^{2}\right)$, como el indicador más aceptado para evaluar su magnitud en la población infantil ${ }^{7}$ ya sea en el ámbito clínico como epidemiológico.

Se recomienda el uso de los siguientes valores límites de IMC de 2 a 19 años: ${ }^{8}$

- Puntaje $Z$ entre 1 y 2 o percentilo 85-96: sobrepeso.

- Puntaje $Z \geq 2$ o percentilo $\geq 97$ : obesidad.

- Puntaje $Z \geq 3$ : obesidad grave.

Para los lactantes, se recomienda la evaluación individual con peso/edad y talla/edad.

El objetivo de esta revisión es ofrecer una actualización, desde la perspectiva del médico intensivista, sobre la fisiopatología de la obesidad y las principales comorbilidades relevantes que pueden estar presentes o desarrollarse en el paciente obeso durante su estadía en la Unidad de Cuidados Intensivos (UCI).

\section{Epidemiología}

La prevalencia de sobrepeso y obesidad en niños y adolescentes está aumentando mundialmente y constituye una epidemia. A modo de ejemplo, en Estados Unidos, el 16,5\% 
de los niños en el rango etario entre 6 y 19 años tienen sobrepeso y un $17 \%$ es obeso. ${ }^{9}$

Este aumento de la obesidad infantil también ocurre en países en vías de desarrollo y se triplicó en países como Chile entre la década de los setenta y la de los noventa, del siglo XX. ${ }^{10}$

Recientemente, una revisión sistemática ${ }^{11}$ señaló que entre un $20 \%$ y un $25 \%$ de los niños latinoamericanos (0-19 años) padecían sobrepeso u obesidad.

Los resultados de la Encuesta Nacional de Nutrición y Salud (ENNyS) demostraron una prevalencia de obesidad de $10,4 \%$ en niños de 6-60 meses. Asimismo, la frecuencia de sobrepeso y obesidad fue creciente con la edad y alcanzó un $20 \%$. Además, esto es variable según la región geográfica y las condiciones socioeconómicas. Esta situación se observa en coexistencia con condiciones de déficit. ${ }^{12}$

Recientemente, se comunicó, en Argentina, para una población de adolescentes de entre 13 y 15 años, que, en un lustro, la prevalencia de sobrepeso había aumentado de $24,5 \%$ a $28,6 \%$ y la obesidad, de $4,4 \%$ a 5,9\%. ${ }^{13}$ Además, en el mismo país, se ha descrito, en niños de 4 años, un $45 \%$ de sobrepeso $\mathrm{u}$ obesidad y un elevado porcentaje de ellos con alteración en el patrón de distribución de grasa corporal. ${ }^{14}$

Del mismo modo, en Chile, en un estudio prospectivo de niños de entre 2 y 18 años de edad, se describió que un 13,9\% presentaban sobrepeso y un $12 \%$, obesidad, sin diferencias por sexo ni grupo etario. ${ }^{15}$

La obesidad es de origen multifactorial y correlaciona genes y estilos de vida. ${ }^{16,17}$ Algunos factores, como el mayor consumo de alimentos procesados y bebidas azucaradas junto con una vida sedentaria, colaborarían en el incremento observado. $^{18}$

En un reciente estudio poblacional europeo constituido por 3000 niños, desde el nacimiento hasta los 5 años, se evaluó la asociación entre la susceptibilidad genética y la obesidad. Se identificó la asociación de variantes genotípicas con la magnitud del crecimiento y composición corporal desde el primer año de vida, pero no en la etapa prenatal. ${ }^{19}$ Estos hallazgos sugieren una mayor susceptibilidad de desarrollar obesidad en la adultez.

Finalmente, las complicaciones de la obesidad en la infancia no solo se manifiestan en la vida adulta como enfermedades crónicas, sino que tienen consecuencias tempranas, tales como hipertensión arterial ${ }^{20}$ hiperlipemia, ${ }^{21}$ apnea obstructiva del sueño (AOS $)^{22}$ y deterioro de su condición de asmático. ${ }^{23}$

Dado que la frecuencia de niños obesos aumenta en la población general y debido a su asociación con comorbilidades, también se ha elevado la prevalencia de niños obesos en unidades de internación y de cuidados intensivos. Se ha descrito en forma concomitante una elevada prevalencia de niños obesos hospitalizados, que alcanzaron hasta un $20 \% .^{24}$

\section{Fisiopatología de la obesidad. Biología de la leptina}

La obesidad es una enfermedad multifactorial en la que numerosos mecanismos fisiopatológicos están implicados, ya sea en el control del peso como en el gasto energético y en sus comorbilidades.

Entre las hormonas adipocitarias, denominadas adipoquininas, la leptina es una hormona de 167 aminoácidos, similar a las citoquinas, sintetizada principalmente por los adipocitos del tejido adiposo blanco y codificada en el gen Lep, localizado en el cromosoma $7 q 31.3 .{ }^{25-27}$ Una vez que es secretada y alcanza la circulación sistémica (en proporción a la magnitud del depósito graso corporal), se une a receptores específicos de superficie celular y activa vías de señalización intracelular. ${ }^{28} \mathrm{El}$ gen del receptor de leptina (LepR) pertenece a la familia de receptores de citoquinas tipo I y codifica seis isoformas distintas del receptor. ${ }^{28}$ Solo LepRb posee los elementos estructurales para la transducción de señales intracelulares. ${ }^{26,29}$ Este se expresa en distintos grupos neuronales del sistema nervioso central (SNC), principalmente el hipotálamo, en los que regula la expresión de neuropéptidos involucrados en el ajuste de la conducta alimentaria, además de la termogénesis y la fertilidad. A nivel periférico, afecta de modo directo el metabolismo y la función en los adipocitos, el pulmón, el riñón, los músculos, el esqueleto, el hígado, la corteza adrenal, las células $\beta$ pancreáticas, entre otros.

La obesidad en niños se correlaciona con niveles circulantes aumentados de leptina. ${ }^{30,31}$ Esta ejerce un rol inmunomodulador ${ }^{32}$ y se desarrolla, en estos pacientes, un estado inflamatorio crónico de bajo grado, ${ }^{33}$ el cual está asociado a infiltración macrofágica del tejido adiposo. Esto determina niveles aumentados de citoquinas proinflamatorias, tales como factor de necrosis tumoral- $\alpha$, interleuquina $1 \beta$, interleuquina- 6 , lo que contribuye al desarrollo de resistencia a la 
insulina ${ }^{34} \mathrm{y}$ disminución de la reserva inmune y metabólica del paciente. ${ }^{35,36}$ De esta manera, la resistencia insulínica, la hipercoagulabilidad y la inflamación caracterizan la obesidad como una afección semejante a una enfermedad crítica. ${ }^{37}$ Las implicancias clínicas son relevantes al ser la respuesta inflamatoria en el paciente obeso distinta del no obeso.

\section{Comorbilidades en el niño obeso}

$\mathrm{Al}$ igual que en los adultos, diversas afecciones comórbidas, como resistencia a la insulina, síndrome metabólico, hipertensión arterial, dislipidemia y diabetes mellitus tipo 2 (DM tipo 2), son secundarias al aumento de grasa abdominal y, por ello, se asocian a una mayor circunferencia de la cintura y obesidad en pacientes pediátricos. ${ }^{38,39}$ Todas ellas pueden ser diagnosticadas al momento de la admisión a la UCI e influir en el pronóstico del paciente pediátrico críticamente enfermo (Figura 1).
Otra entidad crónica asociada con la obesidad infantil es la enfermedad cardiovascular ateroesclerótica. Se ha demostrado que la hiperleptinemia en la población adolescente está asociada con una menor distensibilidad arterial, la cual es un índice funcional relevante para el proceso ateroesclerótico. ${ }^{40}$ La hiperleptinemia es un factor independiente de riesgo cardiovascular, entre otros, de riesgo ateroesclerótico coronario. Es un mediador de la disfunción endotelial y de la hiperplasia neointimal y tiene un efecto paracrino desde el tejido adiposo perivascular sobre el músculo liso vascular. ${ }^{41}$ En el niño obeso, existe un mayor grosor de la capa íntima/media de la pared arterial; esta condición es un marcador no invasivo de cambios ateroescleróticos precoces. Se ha demostrado su reversibilidad durante la infancia mediante la normalización del IMC. ${ }^{42}$

Con respecto a los datos que evalúan la diferencia de mortalidad entre cohortes obesas

FIgURa 1. Problemas médicos y psicosociales presentes en la obesidad infantojuvenil

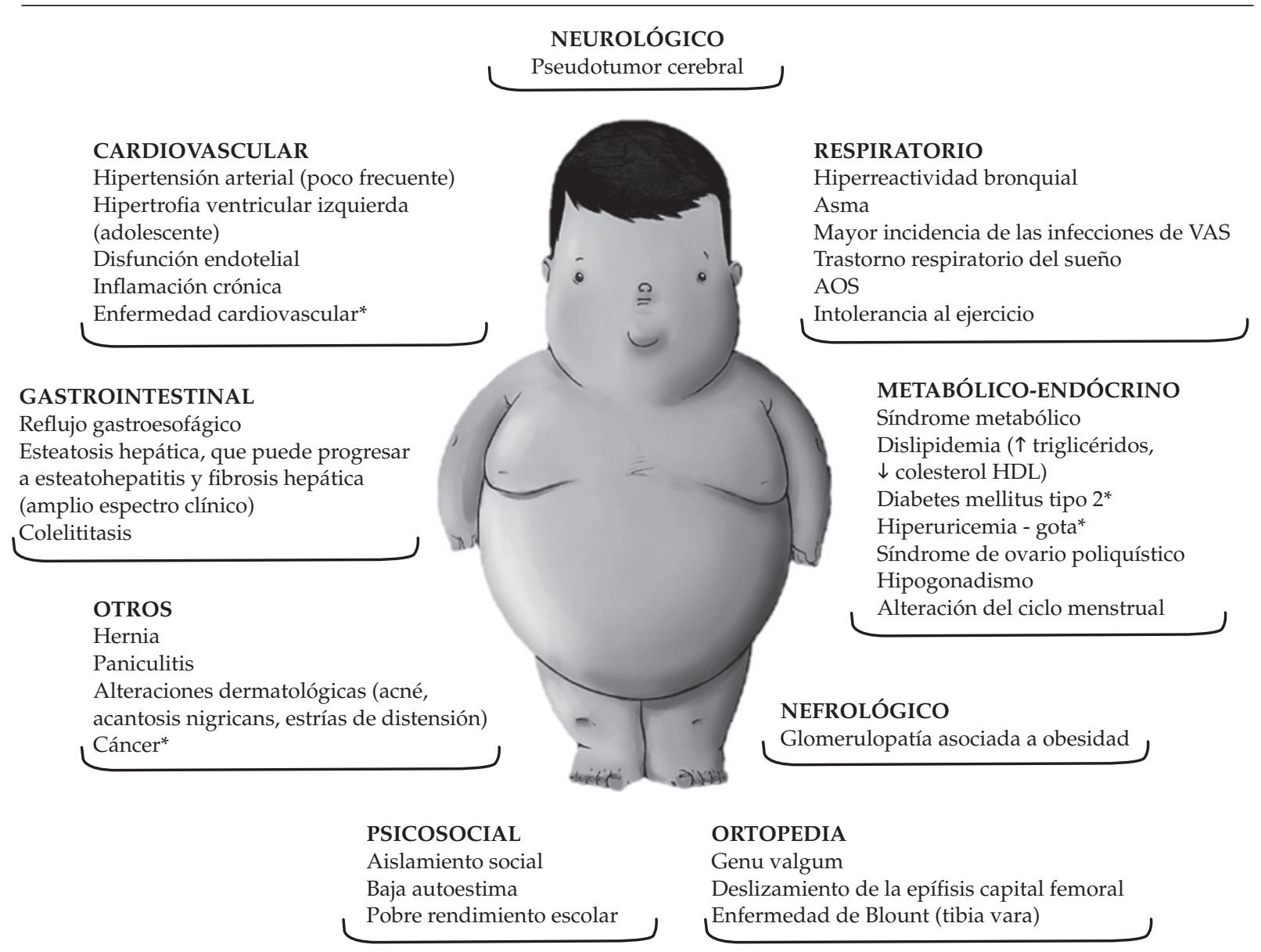

VAS: vía aérea superior; AOS: apnea obstructiva del sueño. * Complicaciones tardías. 
y no obesas, estos no son concluyentes. ${ }^{43}$ En la reciente epidemia de influenza A (H1N1), los niños obesos presentaron una peor evolución. ${ }^{44}$

Una reciente comunicación señaló que el niño obeso que requería ventilación mecánica invasiva no presentaba mayor mortalidad ni días con asistencia ventilatoria mecánica. ${ }^{45}$ Por el contrario, se ha comunicado que el paciente obeso con traumatismo grave ${ }^{46}$ o quemadura ${ }^{47}$ presenta mayor número de complicaciones y días de estadía intra-UCI. Muchas de las complicaciones del paciente traumatizado están relacionadas con enfermedades previas o enfermedad premórbida. ${ }^{46}$

La evidencia sugiere que la obesidad infantil se asocia a una menor sobrevida luego de un paro cardíaco intrahospitalario. ${ }^{48}$ Esto ha planteado evaluar la eficacia de los medicamentos basados de modo exclusivo en el peso corporal. ${ }^{49}$ Así, fármacos altamente solubles en agua y con un pequeño volumen de distribución (epinefrina) pueden alcanzar concentraciones plasmáticas elevadas en el niño obeso y ser potencialmente tóxicos..$^{50}$ En contraparte, medicamentos altamente liposolubles, como la amiodarona, presentan un gran volumen de distribución en el paciente obeso y pueden necesitarse dosis mayores para obtener un efecto comparable. ${ }^{51}$ Se ha recomendado revisar la dosis de desfibrilación empleada ${ }^{52}$ como también de las maniobras estándar de compresión torácica.

El aumento del IMC ocasiona un trabajo cardiovascular, respiratorio y metabólico mayor, que resulta en una disminución significativa de la reserva fisiológica. Se ha demostrado que el comportamiento de esta respuesta presenta una menor eficiencia cardiorrespiratoria en niños obesos, ante pruebas que evalúan el rendimiento aeróbico. ${ }^{53,54}$

En el niño con leucemia linfoblástica aguda, la condición de obeso, independiente de la existencia de predictores de respuesta terapéutica, se ha asociado con un peor pronóstico vital. ${ }^{55}$

Finalmente, en niños críticamente enfermos, aún faltan estudios para determinar el real impacto de la obesidad en la evolución y pronóstico.

\section{Síndrome metabólico}

El sobrepeso y la obesidad están asociados con un aumento en la prevalencia de diversos factores de riesgo cardiovascular. ${ }^{56} \mathrm{El}$ síndrome metabólico (SM) es la suma de diversas anomalías metabólicas, cada una de las cuales es un factor de riesgo independiente de enfermedad cardiovascular y diabetes, y que juntas tienen efecto sinérgico. ${ }^{8}$ Existen diversos criterios de SM en pediatría. Se extrapolan criterios de adultos a niños, ajustados por edad y sexo.

Se recomienda, en la práctica clínica, utilizar el criterio de Cook. ${ }^{57}$ Para el diagnóstico de SM, se deben cumplir tres de los cinco criterios: circunferencia de la cintura $\geq$ Pc 90 , glicemia en ayunas $\geq 110 \mathrm{mg} / \mathrm{dl}$, triglicéridos $\geq 110 \mathrm{mg} / \mathrm{dl}$, colesterol HDL $<40 \mathrm{mg} / \mathrm{dl}$, presión arterial $\geq$ Pc 90 .

El exceso de grasa central (intraabdominal) se relaciona con el desarrollo de enfermedad cardiovascular y DM tipo 2 en la adultez. ${ }^{58}$ Mediante la evaluación de una medida antropométrica, como la razón cintura/estatura, se puede determinar el riesgo cardiometabólico en la población pediátrica. ${ }^{59,60}$

En escolares, se ha demostrado una asociación entre el exceso de peso, la resistencia insulínica y la presencia de SM. ${ }^{6}$ Se ha señalado que entre $30 \%$ y $50 \%$ de los adolescentes obesos presentan $\mathrm{SM}$, comparado con el $7 \%$ con sobrepeso y $0,6 \%$ con IMC en rango normal. 62,63

La hiperglicemia inducida por estrés, frecuente en el paciente críticamente enfermo, puede ser más marcada en el obeso. La hiperglicemia, que inicialmente puede ser beneficiosa (mayor sustrato a tejidos con demandas aumentadas), al prolongarse, puede ocasionar la formación de radicales libres, daño celular y alteración de la inmunidad, lo que resulta en un eventual peor pronóstico. ${ }^{64}$ Sin embargo, no existen datos contundentes sobre la relación del control glicémico en el paciente críticamente enfermo y el pronóstico. ${ }^{65,66}$ Un subgrupo de estos niños puede desarrollar DM tipo 2 con síntomas sutiles, lo cual retarda el diagnóstico. Asimismo, se puede presentar como síndrome hiperosmolar hiperglicémico $(4 \%)$, entidad infrecuente, pero con una significativa morbimortalidad. ${ }^{67-69}$

\section{Hipertensión arterial}

La epidemia mundial de obesidad infantil ha ocasionado que la hipertensión arterial primaria sea una de las causas más frecuentes en la actualidad de hipertensión en niños y adolescentes. ${ }^{70}$

La obesidad es un factor de riesgo independiente para hipertensión arterial en pediatría. ${ }^{71-74} \mathrm{El}$ paciente obeso presenta deterioro funcional del endotelio y músculo liso vascular, ${ }^{73}$ como también disminución de la actividad nerviosa parasimpática. ${ }^{75,76}$ Se ha descrito en niños obesos una mayor masa ventricular izquierda 
asociada a la presencia de hipertensión arterial. ${ }^{74}$ La hipertensión grave y descontrolada puede ocasionar un daño orgánico precoz. ${ }^{77,78}$

\section{Fisiología pulmonar}

Hay datos limitados para la población infantil del impacto de la obesidad en la fisiología pulmonar. Se ha comunicado que las alteraciones encontradas en la función pulmonar más predominantes son la reducción de la capacidad residual funcional (CRF) y el compromiso de la difusión..$^{79}$ Además, el incremento del peso está asociado con una reducción general de los volúmenes pulmonares, lo cual se puede reflejar en un menor estatus funcional. ${ }^{80}$

Recientemente, en un estudio en niños escolares y adolescentes con sobrepeso, en ausencia de síntomas respiratorios, se describió, en un $65 \%$ de ellos, anormalidad de la función pulmonar. La alteración ventilatoria obstructiva y la respuesta positiva a los broncodilatadores fueron las más frecuentes. ${ }^{81}$

En niños, los efectos de la adiposidad en la función pulmonar dependen del sexo y del IMC. ${ }^{82}$

\section{Vía aérea}

El médico intensivista debe poseer entrenamiento en el manejo de la vía aérea, estar preparado para solicitar asistencia y tener acceso al equipo para intubación de la vía aérea difícil. ${ }^{83}$ En una reciente comunicación, se evaluó el impacto de la obesidad como factor de riesgo para el desarrollo de eventos adversos respiratorios durante la sedación en los procedimientos, y fue más frecuente que estos pacientes requirieran intervención en la vía aérea. ${ }^{84}$

Es sabido que los referentes anatómicos clásicos para la vía aérea no son evidentes, por lo que su control constituye un desafío. ${ }^{85}$ Esta se caracteriza por una apertura oral pequeña, con un tejido orofaríngeo redundante, hipertrofia adenoamigdalina y un cuello grueso, corto y con limitada movilidad; también existe infiltración grasa de los músculos, lo que directamente estrecha la vía aérea. Todos estos factores anatómicos pueden hacer que la visualización laringoscópica sea difícil. ${ }^{86,87}$ Además, este escenario puede verse agravado por la dificultad de mantener una vía aérea permeable antes de la intubación endotraqueal, lo dificultoso del uso de la ventilación con máscara facial y una rápida desaturación ocasionada por su menor CRF (presencia de atelectasias en regiones dependientes) ${ }^{88,89}$ lo cual es consecuencia de una reducción del volumen de reserva espiratorio, que altera la capacidad del paciente obeso para tolerar períodos prolongados de apnea ${ }^{90}$ y que, finalmente, ocasiona que este tiempo antes de la desaturación arterial de oxígeno sea breve. ${ }^{91}$

En adultos, ni la obesidad ni el IMC se asociaron con la predicción de una intubación difícil..$^{92}$ El uso de la medición de la circunferencia del cuello en niños es un elemento útil y de fácil acceso para un tamizaje clínico y se ha asociado con el desarrollo de eventos adversos respiratorios. ${ }^{93}$

\section{Asma bronquial}

Se ha descrito que está presente en un 30\% de los pacientes con sobrepeso y obesidad ${ }^{94} \mathrm{y}$ presenta una mayor prevalencia y gravedad en relación con el aumento de peso corporal. . $3,95,96$ Los niveles de leptina sérica se correlacionan positivamente con el IMC y la prevalencia de asma atópico en niños. ${ }^{97}$

En la UCI, el paciente obeso asmático presenta una mayor estadía, pues se recupera más lentamente comparado con el no obeso, con similar gravedad al momento de la admisión. ${ }^{23}$

\section{Sepsis en el paciente obeso}

La obesidad está asociada con disfunción inmune; no obstante, su efecto en la predisposición y pronóstico del paciente séptico no está bien definido. ${ }^{98,99}$ Sin embargo, existe evidencia acumulativa de que el obeso es más propenso a desarrollar complicaciones graves de infecciones comunes. ${ }^{100,101}$

En un modelo experimental en ratones con sepsis por ligadura y punción cecal, se modificó la dieta por un período breve para inducir obesidad y evaluar la respuesta inflamatoria. Se demostró que presentaron una menor probabilidad de supervivencia y mayor daño orgánico, lo que fue dependiente de cambios en el factor nuclear- $\kappa \beta^{102}$ Empero, recientemente, otro modelo experimental de sepsis en un ratón obeso evidenció que la hiperleptinemia mejoró la supervivencia y la respuesta inmune. ${ }^{103}$

\section{Efectos de la obesidad en el riñón}

Estudios observacionales han demostrado que la obesidad es un potencial factor de riesgo para el desarrollo de enfermedades renales de diversas causas, predominantemente enfermedad renal crónica, pero también nefrolitiasis y carcinoma de células renales. ${ }^{104}$ Además, en las últimas décadas, se ha descrito un importante aumento de 
la glomerulopatía asociada a la obesidad, la cual es una patología única ligada al exceso de peso. ${ }^{105}$

Además del conocido vínculo entre SM y desarrollo de DM tipo 2 y enfermedad cardiovascular, ${ }^{58}$ existe evidencia de una importante relación del SM y obesidad con albuminuria y enfermedad renal crónica (ERC), ${ }^{106-108}$ ya presente en forma precoz en personas jóvenes. ${ }^{109}$

Se ha propuesto que el principal motor de la hiperfiltración glomerular existente en el paciente obeso son los adipocitos (Figura 2). Como se ha señalado previamente (vide supra), el tejido adiposo es una fuente de proteínas específicas (leptina, resistina, adiponectina), mediadores inflamatorios, generadores de estrés oxidativo ${ }^{110}$ y componentes del sistema renina-angiotensinaaldosterona (RAA), todos factores que presentan un efecto directo en la enfermedad renal asociada a obesidad. ${ }^{111,112}$ Además, se ha atribuido un papel para la activación del eje RAA a la compresión del hilio renal por la grasa visceral y el aumento de la presión intraabdominal. ${ }^{113}$

La leptina se une a los receptores endoteliales glomerulares y mesangiales, y estimula la proliferación celular (expansión mesangialglomerular), la síntesis de factor de crecimiento transformante $\beta 1$, producción de colágeno tipo I y IV y transporte de glucosa. ${ }^{114}$ Luego, mediante comunicación paracrina entre la célula endotelial y mesangial, la leptina promueve el depósito de

FIGURA 2. Relaciones entre obesidad, insulinorresistencia/hiperinsulinemia compensatoria, componentes de sindrome metabólico y el desarrollo de daño renal, enfermedad renal crónica, enfermedad renal terminal y enfermedad cardiovascular

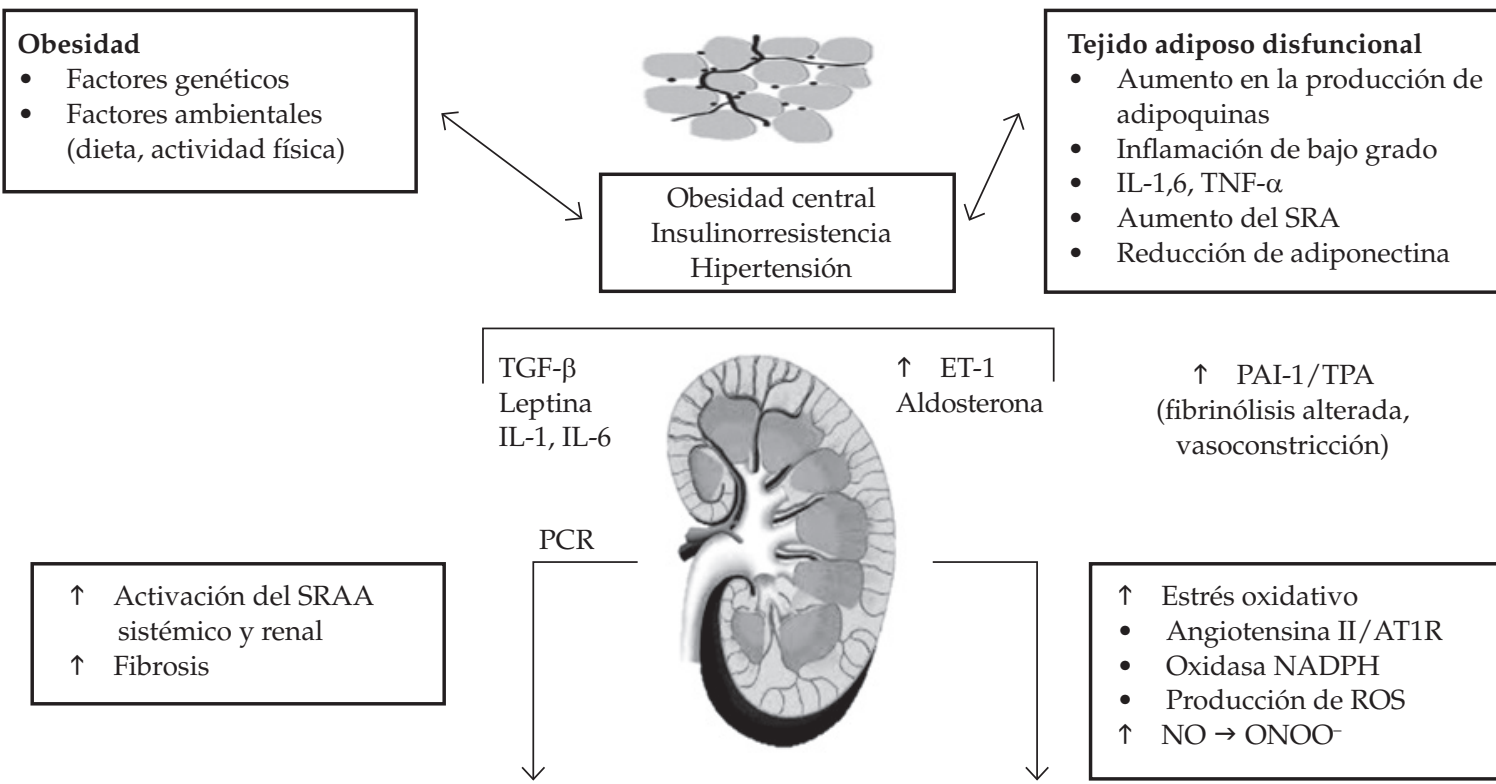

Producción reducida de NO Disfunción endotelial

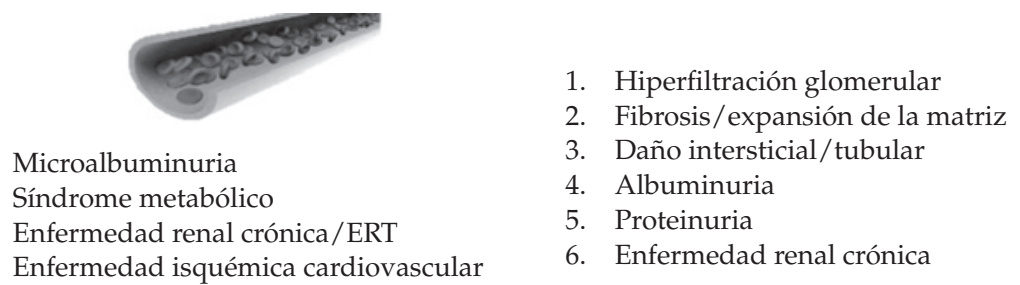

La insulinorresistencia y la hiperinsulinemia compensatoria son el origen de la activación del sistema renina angiotensina (SRA), estrés oxidativo, inflamación sistémica crónica en bajo grado, hipertensión glomerular, microalbuminuria, expansión y fibrosis de la matriz.

AT1R: receptor de la angiotensina II tipo 1; PCR: proteína C reactiva; ERT: enfermedad renal terminal; ET-1: endotelina-1; IL: interleuquina; NADPH: nicotinamida adenina dinucleótido fosfato; NO: óxido nítrico; PAI-1: inhibidor del activador del plasminógeno 1; ROS: especies reactivas de oxígeno; TGF- $\beta$ : factor activador transformante $\beta$; TNF- $\alpha$ : factor de necrosis tumoral $\alpha$; tPA: factor activador del plasminógeno; SRAA: sistema renina-angiotensina-aldosterona; ONOO: peroxinitrito. 
matriz extracelular, lo cual origina, finalmente, glomeruloesclerosis y, en forma concomitante, desarrollo de daño tubulointersticial.

También se debe recordar que el SM ocasiona cambios en la composición urinaria, con incremento en los niveles de ácido úrico, oxalato y calcio, y disminución de los niveles de citrato, lo que influye en la formación de cálculos. ${ }^{115}$

\section{CONCLUSIONES}

El tejido adiposo es un órgano endócrino que produce una variedad de hormonas proteicas. Una de las más abundantes es la leptina, que presenta niveles circulantes aumentados en el niño obeso. La obesidad en el paciente pediátrico tiene importantes efectos fisiopatológicos en diferentes órganos con desarrollo de enfermedades asociadas, tales como inflamación crónica, asma, hipertensión arterial, DM tipo 2 y enfermedad renal crónica.

El paciente pediátrico obeso tiene mayor comorbilidad cuando ingresa por diferentes patologías a una Unidad de Cuidados Intensivos, lo que suma una razón para que la comunidad médica y la sociedad en conjunto brinden medidas eficaces de prevención y tratamiento en familias susceptibles.

\section{REFERENCIAS}

1. Hofmann B. Bariatric surgery for obese children and adolescents: a review of the moral challenges. BMC Med Ethics 2013;14:18.

2. Peirson L, Fitzpatrick-Lewis D, Morrison K, Ciliska D, et al. Prevention of overweight and obesity in children and youth: a systematic review and meta-analysis. CMAJOpen 2015;3(1):E23-33.

3. Mason K, Page L, Balikcioglu PG. Screening for hormonal, monogenic, and syndromic disorders in obese infants and children. Pediatr Ann 2014;43(9):e218-24.

4. Yanovski JA. Pediatric obesity. Rev Endocr Metabol Disord 2001;2(4):371-83.

5. Must A, Phillips SM, Naumova EN. Occurrence and timing of childhood overweight and mortality: findings from the Third Harvard Growth Study. J Pediatr 2012;160(5):743-50.

6. Fontaine KR, Redden DT, Wang C, Westfall AO, et al. Years of life lost due to obesity. JAMA 2003;289(2):187-93.

7. Lobstein T, Baur L, Uauy R. Obesity in children and young people: a crisis in public health. Obes Rev 2004;5(Suppl 1):4-104.

8. Comité Nacional de Nutrición. Guías de práctica clínica para la prevención, el diagnóstico y el tratamiento de la obesidad. Arch Argent Pediatr 2011;109(3):256-66.

9. Ogden CL, Carroll MD, Curtin LR, McDowell MA, et al. Prevalence of overweight and obesity in the United States, 1999-2004. JAMA 2006;295(13):1549-55.

10. Wang Y, Lobstein T. Worldwide trends in childhood overweight and obesity. Int J Pediatr Obes 2006;1(1):11-25.

11. Rivera JÁ, De Cossío TG, Pedraza LS, Aburto TC, et al. Childhood and adolescent overweight and obesity in Latin
America: a systematic review. Lancet Diabetes Endocrinol 2014;2(4):321-32.

12. Argentina. Ministerio de Salud. Encuesta Nacional de Nutrición y Salud. Documento de resultados 2007. [Acceso: 20 de agosto de 2015]. Disponible en http:/ /www.msal. gov.ar/images/stories/bes/graficos/0000000257cnt-a08ennys-documento-de-resultados-2007.pdf.

13. Ferrante D, Linetzky B, Ponce M, Goldberg L, et al. Prevalencia de sobrepeso, obesidad, actividad física y tabaquismo en adolescentes argentinos: Encuestas Mundiales de Salud Escolar y de Tabaco en Jóvenes, 20072012. Arch Argent Pediatr 2014;112(6):496-503.

14. Berghtein IR. Obesidad y sedentarismo en niños de 4 años que asisten a dos jardines de infantes de la ciudad de Río Grande, Tierra del Fuego, Argentina. Arch Argent Pediatr 2014;112(6):557-61.

15. Eyzaguirre F, Mericq V, Ceresa S, Youlton R, et al. Prevalencia de sobrepeso y obesidad en niños que se controlan en pediatría ambulatoria en Clínica Las Condes. Rev Chil Pediatr 2005;76(2):143-9.

16. Poston L. Maternal obesity, gestational weight gain and diet as determinants of offspring long term health. Best Pract Res Clin Endocrinol Metab 2012;26(5):627-39.

17. Öst A, Lempradl A, Casas E, Weigert M, et al. Paternal diet defines offspring chromatin state and intergenerational obesity. Cell 2014;159(6):1352-64.

18. McAllister EJ, Dhurandhar NV, Keith SW, Aronne LJ, et al. Ten putative contributors to the obesity epidemic. Crit Rev Food Sci Nutr 2009;49(10):868-913.

19. Elks CE,Heude B, DeZegher F, BartonSJ, et al. Associations between genetic obesity susceptibility and early postnatal fat and lean mass: an individual participant meta-analysis. JAMA Pediatr 2014;168(12):1122-30.

20. Ahern D, Dixon E. Pediatric hypertension: a growing problem. Prim Care 2015;42(1):143-50.

21. Freedman DS, Dietz WH, Srinivasan SR, Berenson GS. The relation of overweight to cardiovascular risk factors among children and adolescents: the Bogalusa HeartStudy. Pediatrics 1999;103(6 Pt 1):1175-82.

22. Canapari CA, Hoppin AG, Kinane TB, Thomas BJ, et al. Relationship between sleep apnea, fat distribution, and insulin resistance in obese children. J Clin Sleep Med 2011;7(3):268-73.

23. Carroll CL, Bhandari A, Zucker AR, Schramm CM. Childhood obesity increases duration of therapy during severe asthma exacerbations. Pediatr Crit Care Med 2006;7(6):527-31.

24. Woo JG, Zeller MH, Wilson K, Inge T. Obesity identified by discharge ICD-9 codes underestimates the true prevalence of obesity in hospitalized children. J Pediatr 2009;154(3):327-31.

25. Green ED, Maffei M, Braden VV, Proenca R, et al. The human obese $(\mathrm{OB})$ gene: RNA expression pattern and mapping on the physical, cytogenetic, and genetic maps of chromosome 7. Genome Res 1995;5(1):5-12.

26. Friedman JM, Halaas JL. Leptin and the regulation of body weight in mammals. Nature 1998;395(6704):763-70.

27. Zhang Y, Proenca R, Maffei M, Barone M, et al. Positional cloning of the mouse obese gene and its human homologue. Nature 1994;372(6505):425-32.

28. Tartaglia LA, Dembski M, Weng $X$, Deng $N$, et al. Identification and expression cloning of a leptin receptor, OB-R. Cell 1995;83(7):1263-71.

29. Tartaglia LA. The leptin receptor. J Biol Chem 1997;272(10): 6093-6. 
30. Reiterer EE, Sudi KM, Mayer A, Limbert-Zinterl C, et al. Changes in leptin, insulin and body composition in obese children during a weight reduction program. J Pediatr Endocrinol Metab 1999;12(6):853-62.

31. Kelly AS, Metzig AM, Schwarzenberg SJ, Norris AL, et al. Hyperleptinemia and hypoadiponectinemia in extreme pediatric obesity. Metab Syndr Relat Disord 2012;10(2):123-7.

32. Ozata M, Ozdemir IC, Licinio J. Human leptin deficiency caused by a missense mutation: multiple endocrine defects, decreased sympathetic tone, and immune system dysfunction indicate new targets for leptin action, greater central than peripheral resistance to the effects of leptin, and spontaneous correction of leptin-mediated defects. J Clin Endocrinol Metab 1999;84(10):3686-95.

33. Valle M, Martos R, Gascón F, Cañete R, et al. Low-grade systemic inflammation, hypoadiponectinemia and a high concentration of leptin are present in very young obese children, and correlate with metabolic syndrome. Diabetes Metab 2005;31(1):55-62.

34. Steinberger J,Steffen L, Jacobs DRJr, Moran A, etal. Relation of leptin to insulin resistance syndrome in children. Obes Res 2003;11(9):1124-30.

35. XuH, Barnes GT, Yang Q, Tan G, etal. Chronicinflammation in fat plays a crucial role in the development of obesityrelated insulin resistance. J Clin Invest 2003;112(12):1821-30.

36. Wellen KE,HotamisligilGS. Obesity-induced inflammatory changes in adipose tissue. J Clin Invest 2003;112(12):1785-8.

37. Pieracci FM, Barie PS, Pomp A. Critical care of the bariatric patient. Crit Care Med 2006;34(6):1796-804.

38. LeeS,BachaF, GungorN, ArslanianSA. Waistcircumference is an independent predictor of insulin resistance in black and white youths. J Pediatr 2006;148(2):188-94.

39. Sinha R, Fisch G, Teague B, Tamborlane WV, et al. Prevalence of impaired glucose tolerance among children and adolescents with marked obesity. $N$ Engl J Med 2002;346(11):802-10.

40. Singhal A, Farooqi IS, Cole TJ, O'Rahilly S, et al. Influence of leptin on arterial distensibility: a novel link between obesity and cardiovascular disease? Circulation 2002;106(15):1919-24.

41. Payne GA, Tune JD, Knudson JD. Leptin-induced endothelial dysfunction: a target for therapeutic interventions. Curr Pharm Des 2014;20(4):603-8.

42. Wunsch R, De Sousa G, Toschke AM, Reinehr T. Intimamedia thickness in obese children before and after weight loss. Pediatrics 2006;118(6):2334-40.

43. NevilleAL, BrownCV,WengJ,Demetriades D, etal.Obesity is an independent risk factor of mortality in severely injured blunt trauma patients. Arch Surg 2004;139(9):983-7.

44. Van Kerkhove MD, Vandemaele KA, Shinde V, JaramilloGutierrezG, etal. Risk factors for severe outcomes following 2009 influenza A(H1N1) infection: a global pooled analysis. PloS Med 2011;8(7):e1001053.

45. Goh VL, Wakeham MK, Brazauskas R, Mikhailov TA, et al. Obesity is not associated with increased mortality and morbidity in critically ill children. JPEN J Parenter Enteral Nutr 2013;37(1):102-8.

46. Brown CV, Neville AL, Salim A, Rhee P, et al. The impact of obesity on severely injured children and adolescents. J Pediatr Surg 2006;41(1):88-91.

47. Ross E, Burris A, Murphy JT. Obesity and outcomes following burns in the pediatric population. J Pediatr Surg 2014;49(3):469-73.

48. Srinivasan V, Nadkarni VM, Helfaer MA, Carey SM, et al. Childhood obesity and survival after in-hospital pediatric cardiopulmonary resuscitation. Pediatrics 2010;125(3):e481-8.

49. Yamamoto LG, Inaba AS, Young LL, Anderson KM. Improving length-based weightestimates by adding a body habitus (obesity) icon. Am J Emerg Med 2009;27(7):810-5.

50. Fisher DG, Schwartz PH, Davis AL. Pharmacokinetics of exogenous epinephrine in critically ill children. Crit Care Med 1993;21(1):111-7.

51. Steinberg C, Notterman DA. Pharmacokinetics of cardiovascular drugs in children. Inotropes and vasopressors. Clin Pharmacokinet 1994;27(5):345-67.

52. Berg MD, Banville IL, Chapman FW, Walker RG, et al. Attenuating the defibrillation dosage decreases postresuscitation myocardial dysfunction in a swine model of pediatric ventricular fibrillation. Pediatr Crit Care Med 2008;9(4):429-34.

53. López A, Sotomayor L, Álvarez MP, Céspedes P, et al. Rendimiento aeróbico en niños obesos de 6 a 10 años. Rev Chil Pediatr 2009;80(5):444-50.

54. Kain J, Olivares S, Romo M, Leyton B, et al. Estado nutricional y resistencia aeróbica en escolares de educación básica: línea base de un Proyecto de Promoción de la Salud. Rev Med Chil 2004;132(11):1395-402.

55. Orgel E, Tucci J, Alhushki W, Malvar J, et al. Obesity is associated with residual leukemia following induction therapy for childhood B-precursor acute lymphoblastic leukemia. Blood 2014;124(26):3932-8.

56. Ford ES, Mokdad AH, Ajani UA. Trends in risk factors for cardiovascular disease among children and adolescents in the United States. Pediatrics 2004;114(6):1534-44.

57. Cook S, Auinger P, Huang TT. Growth curves for cardiometabolic risk factors in children and adolescents. J Pediatr 2009;155(3):S6.e15-26.

58. Juonala M, Magnussen CG, Berenson GS, Venn A, et al. Childhood adiposity, adult adiposity, and cardiovascular risk factors. N Engl J Med 2011;365(20):1876-85.

59. Khoury M, Manlhiot C, McCrindle BW. Role of the waist/ height ratio in the cardiometabolic risk assessment of children classified by body mass index. J Am Coll Cardiol 2013;62(8):742-51.

60. Arnaiz P, Grob F, Cavada G, Domínguez A, et al. La razón cintura estatura en escolares no varía con el género, la edad ni la maduración puberal. Rev Med Chil 2014;142(5):574-8.

61. Mardones F, Arnaiz P, Barja S, Giadach C, et al. Estado nutricional, síndrome metabólico y resistencia a la insulina en niños deSantiago, Chile. Nutr Hosp 2013;28(6):1999-2005.

62. Cook S, Weitzman M, Auinger P, Nguyen M, et al. Prevalence of a metabolic syndrome phenotype in adolescents: findings from the third National Health and Nutrition Examination Survey, 1988-1994. Arch Pediatr Adolesc Med 2003;157(8):821-7.

63. Weiss R, Dziura J, Burgert TS, TamborlaneWV, etal.Obesity and the metabolic syndrome in children and adolescents. N Engl J Med 2004;350(23):2362-74.

64. Clark L, Preissig C, Rigby MR, Bowyer F. Endocrine issues in the pediatric intensive care unit. Pediatr Clin North Am 2008;55(3):805-33.

65. Tiruvoipati R, Chiezey B, Lewis D, Ong K, et al. Stress hyperglycemia may not be harmful in critically ill patients with sepsis. J Crit Care 2012;27(2):153-8.

66. Branco RG, Xavier L, Garcia PC, Piva JP, et al. Prospective operationalization and feasibility of a glycemic control protocol in critically ill children. Pediatr Crit Care Med 2011;12(3):265-70.

67. Carchman RM, Dechert-Zeger M, Calikoglu AS, Harris 
BD. A new challenge in pediatric obesity: pediatric hyperglycemic hyperosmolar syndrome. Pediatr Crit Care Med 2005;6(1):20-4

68. Lin JJ, McKenney DW, Price C, Morrison RR, et al. Continuous venovenous hemodiafiltration in hypernatremic hyperglycemic nonketotic coma. Pediatr Nephrol 2002;17(11):969-73.

69. American Diabetes Association. Hyperglycemic crises in patients with diabetes mellitus. Diabetes Care 2001;24(11):1988-96.

70. Flynn J. The changing face of pediatric hypertension in the era of the childhood obesity epidemic. Pediatr Nephrol 2013;28(7):1059-66.

71. McNiece KL, Poffenbarger TS, Turner JL, Franco KD, et al. Prevalence of hypertension and pre-hypertension among adolescents. J Pediatr 2007;150(6):640-4.

72. GundogduZ. Relationship between BMIand blood pressure in girls and boys. Public Health Nutr 2008;11(10):1085-8.

73. Aggoun Y, Farpour-Lambert NJ, Marchand LM, Golay E, et al. Impaired endothelial and smooth muscle functions and arterialstiffness appear before puberty in obese children and are associated with elevated ambulatory blood pressure. Eur Heart J 2008;29(6):792-9.

74. Maggio AB, Aggoun Y, Marchand LM, Martin XE, et al. Associations among obesity, blood pressure, and left ventricular mass. J Pediatr 2008;152(4):489-93.

75. Rabbia F, Silke B, Conterno A, Grosso T, et al. Assessment of cardiac autonomic modulation during adolescent obesity. Obes Res 2003;11(4):541-8.

76. Feber J, Ruzicka M, Geier P, Litwin M. Autonomic nervous system dysregulation in pediatric hypertension. Curr Hypertens Rep 2014;16(5):426.

77. Singh D, Akingbola O, Yosypiv I, El-Dahr S. Emergency management of hypertension in children. Int J Nephrol 2012;2012:420247.

78. Constantine E, Merritt C. Hypertensive emergencies in children: identification and management of dangerously high blood pressure. Minerva Pediatr 2009;61(2):175-84.

79. Li AM, Chan D, Wong E, Yin J, et al. The effects of obesity on pulmonary function. Arch Dis Child 2003;88(4):361-3.

80. Davidson WJ, Mackenzie-Rife KA, Witmans MB, Montgomery MD, et al. Obesity negatively impacts lung function in children and adolescents. Pediatr Pulmonol 2014;49(10):1003-10.

81. Assuncao SN, Daltro $\mathrm{CH}$, Boa Sorte NC, Ribeiro Júnior $\mathrm{Hda}$, et al. Lung function in the absence of respiratory symptoms in overweight children and adolescents. J Bras Pneumol 2014;40(2):134-41.

82. Khan S, Little J, Chen Y. Relationship between adiposity and pulmonary function in school-aged Canadian children. Pediatr Allergy Immunol Pulmonol 2014;27(3):126-32.

83. Bailey KA. Special considerations in the critically ill morbidly obese child. Crit Care Clin 2010;26(4):699-702.

84. Scherrer PD, Mallory MM, Cravero JP, Lowrie L, et al. The impact of obesity on pediatric procedural sedation-related outcomes: results from the Pediatric Sedation Research Consortium. Paediatr Anaesth 2015;25(7):689-97.

85. Ray RM, Senders CW. Airway management in the obese child. Pediatr Clin Noth Am 2001;48(4):1055-63.

86. Malhotra A, Hillman D. Obesity and the lung: 3. Obesity, respiration and intensive care. Thorax 2008;63(10):925-31.

87. DeaneS, Thomson A. Obesity and the pulmonologist. Arch Dis Child 2006;91(2):188-91.

88. Tait AR, Voepel-Lewis T, Burke C, Kostrzewa A, et al. Incidence and risk factors for perioperative adverse respiratory events in children who are obese. Anesthesiology 2008;108(3):375-80.

89. Nafiu OO, Reynolds PI, Bamgbade OA, Tremper KK, et al. Childhood body mass index and perioperative complications. Paediatr Anaesth 2007;17(5):426-30.

90. El-Solh AA. Clinical approach to the critically ill, morbidly obese patient. Am J Respir Crit Care Med 2004;169(5):557-61.

91. Juvin P, Lavaut E, Dupont H, Lefevre P, et al. Difficult tracheal intubation is more common in obese than in lean patients. Anesth Analg 2003;97(2):595-600.

92. Brodsky JB, Lemmens HJ, Brock-Utne JG, Vierra M, et al. Morbid obesity and tracheal intubation. Anesth Analg 2002;94(3):732-6.

93. Nafiu OO, Burke CC, Gupta R, Christensen R, et al. Association of neck circumference with perioperative adverse respiratory events in children. Pediatrics 2011;127(5):e1198-205.

94. Lang JE, Feng H, Lima JJ. Body mass index-percentile and diagnostic accuracy of childhood asthma. J Asthma 2009;46(3):291-9.

95. Carroll CL, Uygungil B, Zucker AR, Schramm CM. Identifying an at-risk population of children with recurrent near-fatal asthma exacerbations. J Asthma 2010;47(4):460-4.

96. Carroll CL, Stoltz P, Raykov N, Smith SR, et al. Childhood overweight increases hospital admission rates for asthma. Pediatrics 2007;120(4):734-40.

97. Guler N, Kirerleri E, Ones U, Tamay Z, et al. Leptin: does it have any role in childhood asthma? J Allergy Clin Immunol 2004;114(2):254-9.

98. Arabi YM, Dara SI, Tamim HM, Rishu AH, et al. Clinical characteristics, sepsis interventions and outcomes in the obese patients with septic shock: an international multicenter cohort study. Crit Care 2013;17(2):R72.

99. Blanco-Quirós A, Casado-Flores J, Arranz E, Garrote JA, et al. Influence of leptin levels and body weight in survival of children with sepsis. Acta Paediatr 2002;91(6):626-31.

100.Falagas ME, Kompoti M. Obesity and infection. Lancet Infect Dis 2006;6(7):438-46.

101.Wang HE, Griffin R, Judd S, Shapiro NI, et al. Obesity and risk of sepsis: a population-based cohort study. Obesity (Silver Spring) 2013;21(12):E762-9.

102.Kaplan JM, Nowell M, Lahni P, O'Connor MP, et al. Short-term high fat feeding increases organ injury and mortality after polymicrobial sepsis. Obesity (Silver Spring) 2012;20(10):1995-2002.

103.Sieg1D, Annecke T, Johnson BL 3rd, Schlag C, et al. Obesityinduced hyperleptinemia improves survival and immune response in a murine model of sepsis. Anesthesiology 2014;121(1):98-114.

104.Wang Y, Chen X, Song Y, Caballero B, et al. Association between obesity and kidney disease: a systematic review and meta-analysis. Kidney Int 2008;73(1):19-33.

105.Kambham N, MarkowitzGS, Valeri AM, Lin J, etal. Obesityrelated glomerulopathy: an emerging epidemic. Kidney Int 2001;59(4):1498-509.

106.Iseki K, Ikemiya Y, Kinjo K, Inoue T, et al. Body mass index and the risk of development of end-stage renal disease in a screened cohort. Kidney Int 2004;65(5):1870-6.

107.Sowers JR. Metabolic risk factors and renal disease. Kidney Int 2007;71(8):719-20.

108.Ejerblad E, Fored CM, Lindblad P, Fryzek J, et al. Obesity and risk for chronic renal failure. J Am Soc Nephrol 2006;17(6):1695-702.

109.Tomaszewski M, Charchar FJ, Maric C, McClure J, et al. Glomerular hyperfiltration: a new marker of metabolic 
risk. Kidney Int 2007;71(8):816-21.

110.Kelly AS, Steinberger J, Kaiser DR, Olson TP, et al. Oxidative stress and adverse adipokine profile characterize the metabolic syndrome in children. J Cardiometab Syndr 2006;1(4):248-52.

111. Alicic RZ, Patakoti R, Tuttle KR. Direct and indirect effects of obesity on the kidney. Adv Chronic Kidney Dis 2013;20(2):121-7.

112. Whaley-Connell AT, Chowdhury NA,HaydenMR,StumpCS, et al. Oxidative stress and glomerular filtration barrier injury: role of the renin-angiotensin system in the Ren2 transgenic rat. Am J Physiol Renal Physiol 2006;291(6):F1308-14.

113.Felizardo RJ, da Silva MB, Aguiar CF, Câmara NO. Obesity in kidney disease: a heavyweight opponent. World JNephrol 2014;3(3):50-63.

114.Wolf G, Hamann A, Han DC, Helmchen U, et al. Leptin stimulates proliferation and TGF-beta expression in renal glomerular endothelial cells: potential role in glomerulosclerosis. Kidney Int 1999;56(3):860-72.

115. GorbachinskyI,AkpinarH,AssimosDG. Metabolicsyndrome and urologic diseases. Rev Urol 2010;12(4):e157-80.

Los siguientes resúmenes y comentarios de trabajos seleccionados se encuentran disponibles en la versión electrónica de este número.

N ENGL J MED 2015;373:1497-506

Administración temprana de budesonide inhalado para la prevención de displasia broncopulmonar (Bassler D, et al. Early inhaled budesonide for the prevention of bronchopulmonary dysplasia)

Comentario: Dr. José M. Ceriani Cernadas. Servicio de Neonatología. Hospital Italiano de Buenos Aires.

PEDIATRICS 2015;136(4):635-41

Fuente de infección por pertussis en lactantes en EE. UU. (Skoff T, et al. Sources of infant pertussis infection in the United States)

Comentario: Dra. Ángela Gentile. Hospital de Niños Ricardo Gutiérrez. Buenos Aires.

PEDIATRICS 2015;136(4)

Experiencias tempranas en la carrera de pediatras que efectuaron o no un programa de postgrado (Byrne BJ, et al. Early career experiences of pediatricians pursuing or not pursuing fellowship training) Comentario: Dra. Claudia María Palladino. Secretaria de Filiales y Regiones CD SAP. Directora Provincial Materno Infantil Catamarca.

PEDIATRICS 2016;137(1):1-9

Expansión de volumen precoz y evolución del síndrome urémico hemolítico (Ardissino G, et al. Early volume expansion and outcomes of hemolytic uremic syndrome)

Comentario: Dr. Alejandro Balestracci. Unidad de Nefrología. Hospital de Niños Pedro de Elizalde. Buenos Aires.

LANCET 2015; 386:1041-48

Objetivos de saturación de oxígeno en lactantes con bronquiolitis (BIDS): un estudio de equivalencia, aleatorizado, doble ciego (Cunningham $S$, et al. Oxygen saturation targets in infants with bronchiolitis (BIDS): a double-blind, randomised, equivalence trial)

Comentario: Dra. Hilda Giugno. Servicio de Neumonología. Hospital de Pediatría JP Garrahan. Buenos Aires.

PEDIATRIC PULMONOLOGY 2015;50:1025-32

Eficacia de la profilaxis con palivizumab en niños con cardiopatía congénita: un estudio de casos y controles (Pzyurt A, et al. Efficacy of palivizumab prophylaxis among infants ith congenital heart disease: A case control study)

Comentario: Dra. Gabriela Bauer. Seguimiento de RN de Alto Riesgo. Hospital de Pediatría "Prof. Dr. Juan P. Garrahan". Buenos Aires. 